\title{
PROCESS AND SYSTEMS Predictors of 30-day hospital readmission: The direct comparison of number of discharge medications to the HOSPITAL score and LACE index
}

\author{
Authors: Robert Robinson, ${ }^{A}$ Mukul Bhattarai, ${ }^{B}$ Tamer Hudali ${ }^{C}$ and Carrie Vogler ${ }^{D}$
}

Effective hospital readmission risk prediction tools exist, but do not identify actionable items that could be modified to reduce the risk of readmission. Polypharmacy has attracted attention as a potentially modifiable risk factor for readmission, showing promise in a retrospective study. Polypharmacy is a very complex issue, reflecting comorbidities and healthcare resource utilisation patterns. This investigation compares the predictive ability of polypharmacy alone to the validated HOSPITAL score and LACE index readmission risk assessment tools for all adult admissions to an academic hospitalist service at a moderate sized university-affiliated hospital in the American Midwest over a 2-year period. These results indicate that the number of discharge medications alone is not a useful tool in identifying patients at high risk of hospital readmission within 30 days of discharge. Further research is needed to explore the impact of polypharmacy as a risk predictor for hospital readmission.

KEYWORDS: Readmission, discharge medications, LACE index, HOSPITAL score, polypharmacy

\section{Introduction}

Hospital readmissions are common and expensive, with nearly $20 \%$ of Medicare patients being readmitted to a hospital within 30 days of discharge at an overall cost of nearly US $\$ 20$ billion per year. ${ }^{1}$ Because of this high frequency and cost, hospital readmissions are a target for healthcare cost savings in the Medicare Hospital Readmission Reduction Program (HRRP). Therefore, healthcare organisations are investing considerable resources into efforts to reduce hospital readmission.

Authors: A associate professor and academic hospitalist, Southern Illinois University School of Medicine, Springfield, USA; ${ }^{B}$ cardiology fellow, Southern Illinois University School of Medicine, Springfield, USA; ' pulmonary critical care medicine fellow, University of Alabama at Birmingham, Birmingham, USA; ${ }^{\text {D }}$ clinical associate professor, Southern Illinois University Edwardsville School of Pharmacy, Edwardsville, USA and adjunct clinical assistant professor, Southern Illinois University School of Medicine, Springfield, USA
The underlying risk factors for hospital readmission are diverse. Studies have identified age, ethnicity, lack of outpatient healthcare provider, major surgery, medical comorbidities, length of hospitalisation, previous admissions, failure to transfer important information to the outpatient setting, early discharge and the number of discharge medications etc are associated with hospital readmission within 30 days. ${ }^{2-5}$ However, clinicians still struggle to predict the patients who are at high risk of hospital readmission. ${ }^{6}$

Readmission risk assessment can be accomplished with a variety of assessment tools that range from multidisciplinary patient interviews to simple screening tools using a handful of variables. $^{4,7-9}$

The easy to use HOSPITAL score is one such screening tool. ${ }^{10}$ The HOSPITAL score uses seven readily available clinical predictors (haemoglobin level at discharge, discharge from oncology, sodium level at discharge, procedure during hospitalisation, index admission type, number of hospital admissions and length of stay) to accurately identify patients at high risk of potentially avoidable hospital readmission within 30 days (Table 1). This score has been internationally validated in a population of over 100,000 patients at large academic medical centres (average size of 975 beds) and

\section{Table 1. HOSPITAL score}

\begin{tabular}{|c|c|}
\hline Attribute & $\begin{array}{l}\text { Points if } \\
\text { positive }\end{array}$ \\
\hline Low haemoglobin at discharge $(<12 \mathrm{~g} / \mathrm{dL})$ & 1 \\
\hline Discharge from an oncology service & 2 \\
\hline Low sodium level at discharge ( $<135 \mathrm{mmol} / \mathrm{L})$ & 1 \\
\hline Procedure during hospital stay (ICD10 coded) & 1 \\
\hline Index admission type urgent or emergent & 1 \\
\hline \multicolumn{2}{|l|}{$\begin{array}{l}\text { Number of hospital admissions during the } \\
\text { previous year: }\end{array}$} \\
\hline $0-1$ & 0 \\
\hline $2-5$ & 2 \\
\hline$>5$ & 5 \\
\hline Length of stay $\geq 5$ days & 2 \\
\hline
\end{tabular}


has been shown to have superior discriminative ability over some prediction tools. $^{8,10,11}$

Another simple prediction model for predicting hospital readmission which uses both administrative and primary data is the LACE index. ${ }^{12}$ The LACE index uses four variables: length of stay, acuity of the admission, comorbidity of the patient and emergency department use in the duration of 6 months before admission. ${ }^{12}$ This model has been internally validated using data collected from 4,812 patients discharged from 11 community hospitals in Ontario, and it was externally validated using administrative data collected randomly from 1,000,000 discharges. ${ }^{12}$ The LACE index has been shown to have moderate discrimination in studies conducted in North America with over 26,000 Medicare admissions, ${ }^{13} 110,000$ discharges in the Chicago, Illinois area ${ }^{14}$ and 600 patients in a community hospital. ${ }^{15}$

Despite this accuracy, these risk identification tools do not identify modifiable risk factors for hospital readmission. ${ }^{10,16,17}$ Identifying and evaluating potentially modifiable risk factors for readmission, such as polypharmacy, is a focus of active investigation. $2,18,19$

The role of polypharmacy as an independent risk factor predicting 30-day readmission is a recent debate. ${ }^{20,21}$ Morandi et al studied the risk of readmission to elderly patients admitted to a rehabilitation facility and found that having a prescription of seven or more medications significantly increased the risk of hospital readmission within 30 days (hazard ratio (HR) 3.94; $95 \%$ confidence interval (CI) 1.62-9.54; $p=0.002$ ). ${ }^{22}$ Another study showed that polypharmacy increases the risk of hospital admission with a dose-response relation (5-9 drugs: odds ratio (OR) 1.18; $95 \%$ CI 1.12-1.24; $\geq 10$ drugs: OR 1.54; $95 \%$ CI 1.42-1.66). ${ }^{23}$

Our study compares the HOSPITAL and LACE readmission scores with number of medications on discharge as predictors for 30-day readmission in a university affiliated hospital in the American Midwest.

\section{Materials and methods}

All eligible adult medical patients discharged from the Southern Illinois University School of Medicine (SIU-SOM) hospitalist service from Memorial Medical Center, a university-affiliated tertiary care centre from 01 January 2015 to 01 January 2017 were studied retrospectively to determine if the number of discharge medications was a significant predictor of all-cause (avoidable and unavoidable) hospital readmission within 30 days. Exclusion criteria included transfer to another acute care hospital, leaving the hospital against medical advice or death. The endpoint of within 30 days of hospital discharge for all-cause readmission was chosen because it is the measure used by the Medicare HRRP.

Data on age, gender, diagnosis related group, International Classification of Disease (ICD) diagnosis codes, emergency department visits in the last 6 months, length of stay, hospital readmission within 30 days, discharge medications, and the other variables in the HOSPITAL score (Table 1) and LACE index (Table 2) were extracted from the electronic health record in a de-identified manner for analysis. Missing laboratory data (haemoglobin and sodium from the day of discharge) were coded to be in the normal range as was done in the international validation study of the HOSPITAL score. ${ }^{11}$

Quantifying medications at the time of hospital discharge was accomplished in the same manner as outlined by Picker and
Table 2. LACE index

\begin{tabular}{|c|c|}
\hline Attribute & Points if positive \\
\hline \multicolumn{2}{|l|}{ Length of stay: } \\
\hline Less than 1 day & 0 \\
\hline 1 day & 1 \\
\hline 2 days & 2 \\
\hline 3 days & 3 \\
\hline 4-6 days & 4 \\
\hline 7-13 days & 5 \\
\hline$\geq 14$ days & 7 \\
\hline Acute or emergent admission & 3 \\
\hline \multicolumn{2}{|l|}{ Charlson comorbidity index score: } \\
\hline 0 & 0 \\
\hline 1 & 1 \\
\hline 2 & 2 \\
\hline 3 & 3 \\
\hline$\geq 4$ & 5 \\
\hline \multicolumn{2}{|c|}{$\begin{array}{l}\text { Visits to emergency department in } \\
\text { previous } 6 \text { months: }\end{array}$} \\
\hline 0 & 0 \\
\hline 1 & 1 \\
\hline 2 & 2 \\
\hline 3 & 3 \\
\hline$\geq 4$ & 4 \\
\hline
\end{tabular}

colleagues by counting all medications prescribed at the time of hospital discharge, excluding over the counter medications or nutritional supplements. ${ }^{2}$

The study hospital does not have a distinct oncology admitting service. To address the increased risk of readmission in oncology patients found in other studies using the HOSPITAL score, this study classified patients with oncology related diagnosis ICD codes to have been discharged from an oncology service. Because data is only available from the study hospital, readmissions at other hospitals were not detected.

Institutional review board review for this study was obtained from the Springfield Committee for Research Involving Human Subjects. This study was determined not to meet the criteria for research involving human subjects according to 45 CFR 46.101 and 45 CFR 46.102.

\section{Statistical analysis}

The number of medications at discharge, the HOSPITAL score and LACE index were investigated as predictors of all-cause hospital readmission within 30 days. Qualitative variables were compared using Pearson $\chi^{2}$ or Fisher's exact test and reported as frequency (\%). Quantitative variables were compared using the non-parametric Mann-Whitney $U$ test and reported as mean \pm standard deviation.

The HOSPITAL score and LACE index were calculated for each admission. HOSPITAL scores of $0-4$ points were classified as 


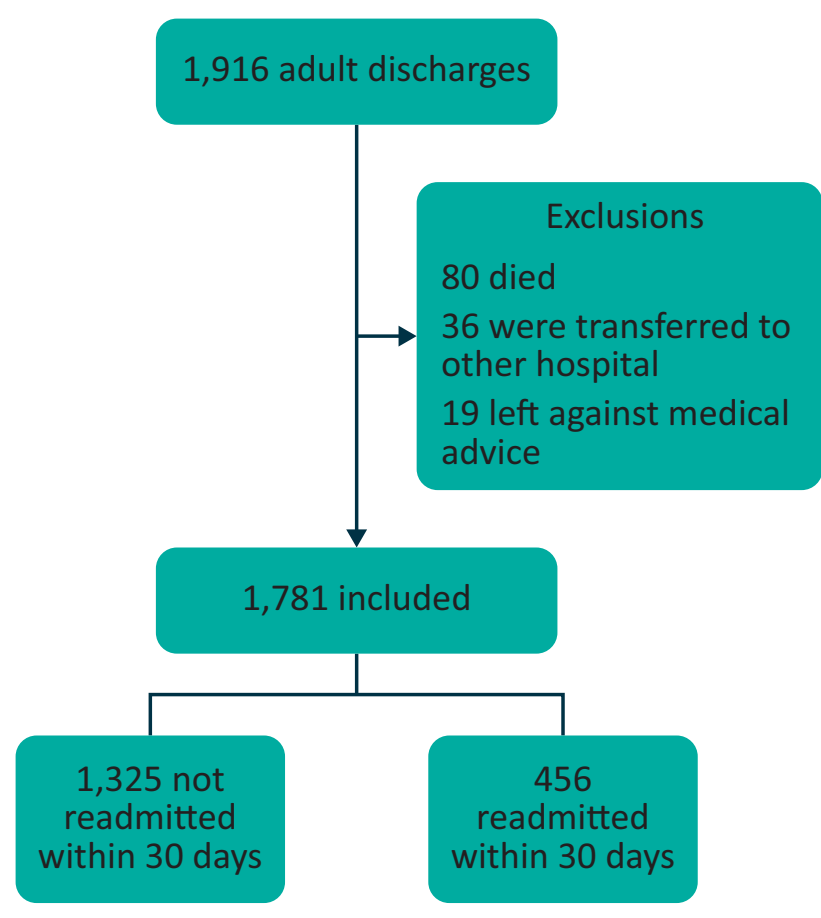

Fig 1. Study flow diagram.

low risk for readmission (5\%), 5-6 points as intermediate risk $(10 \%)$, and $>7$ more points as high risk (20\%) based on the initial validation study of the HOSPITAL score. ${ }^{10}$

LACE indexes ranged from 0-19, with an expected probability for readmission of $0 \%$ to $43.7 \%$ based on the initial validation study of the LACE score. ${ }^{17}$

Discharge medication numbers ranged from 0-12 or more, with expected probabilities of readmission based on the work of Picker and colleagues. ${ }^{2}$

These readmission risk predictions were used to calculate a Brier score to evaluate the accuracy of each predictor. Two sided $p$-values $<0.05$ were considered significant. Variables from univariate analysis with a $p$-value of 0.05 or less were evaluated using multivariate logistic regression with stepwise backward variable selection.

Most statistical analyses were performed using SPSS version 22 (SPSS Inc, Chicago, USA).

The Brier score was calculated with R version 3.4.2 (R Foundation for Statistical Computing, Vienna, Austria).

\section{Results}

During the study period (2 years), 1,916 discharges were recorded for the SIU-SOM hospitalist service. The analysis includes data for the 1,781 discharges for 1,410 individual patients that met inclusion criteria (Fig 1). Of these discharges, 456 (27\%) were readmitted to the same hospital within 30 days. The overall study population was $47 \%$ female, had an average age of 63 years and spent an average of 7.9 days in the hospital.

The patients readmitted within 30 days of discharge were more frequently admitted to the hospital in the last year, evaluated in the emergency department in the last 6 months, had higher HOSPITAL scores, higher LACE index values, higher Charlson

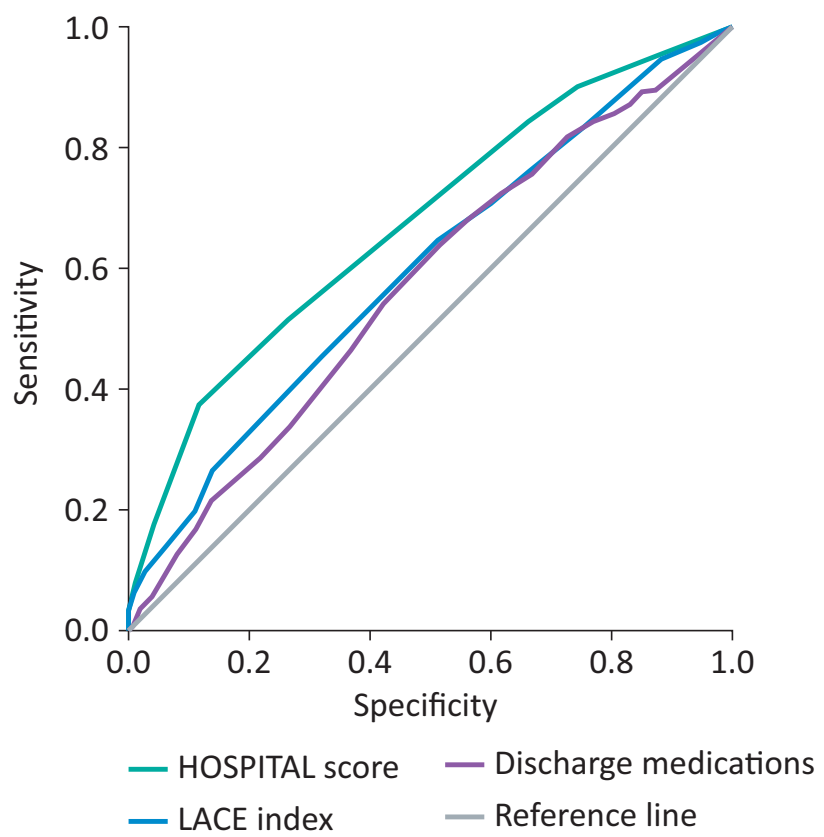

Fig 2. Receiver operating characteristic curves for the number of discharge medications, HOSPITAL score and LACE index.

comorbidity scores, and had more medications at the time of discharge. The medical comorbidities of a history of myocardial infarction, congestive heart failure (CHF), cirrhosis, diabetes and renal disease were seen more frequently in readmitted patients. These differences were statistically significant in univariate analysis (Table 3). Multivariate analysis did not show the number of medications at the time of hospital discharge to be a risk factor for hospital readmission (Table 4).

A receiver operating characteristic evaluation (Fig 2) showed that the HOSPITAL score and LACE index had fair discrimination for hospital readmission in this population. The HOSPITAL score had a C statistic of 0.675 (95\% CI 0.65-0.70), a Brier score of 0.217 , and a Hosmer-Lemeshow goodness of fit test showed a $\chi^{2}$ value of 10.36 with a $p$ value of 0.035 . The LACE index had a $C$ statistic of 0.598 (95\% CI 0.58-0.64), a Brier score of 0.212, and a HosmerLemeshow goodness of fit test showed a $\chi^{2}$ value of 6.15 with a $p$ value of 0.292 . The number of discharge medications had a $C$ statistic of 0.571 (95\% CI $0.54-0.60$ ), a Brier score of 0.200 , and a Hosmer-Lemeshow goodness of fit test showed a $\chi^{2}$ value of 10.13 with a $p$ value of 0.182 .

\section{Discussion}

In the current study, we compared the use of HOSPITAL score, the LACE index and the number of medications upon discharge for predicting all-cause of hospital readmission in our study population. We found that the number of medications prescribed upon hospital discharge is not a significant predictor of hospital readmission in a moderate sized not-for-profit universityaffiliated tertiary care hospital in the American Midwest. The appropriateness of medication therapy should not always be assessed by the number of medications alone. Complex multiple 
Table 3. Baseline characteristics of the study population by 30-day readmission status

\begin{tabular}{|c|c|c|c|}
\hline Characteristic & $\begin{array}{l}\text { Not readmitted within } \\
30 \text { days, } n=1,325\end{array}$ & $\begin{array}{l}\text { Readmitted within } \\
30 \text { days, } n=456\end{array}$ & $\mathrm{p}$ value \\
\hline Age, mean (SD) & $63(16.0)$ & $64(15.5)$ & 0.144 \\
\hline Female, n ( \% ) & $624(47 \%)$ & $216(47 \%)$ & 0.919 \\
\hline Length of stay, days, mean (SD) & $7.7(7.1)$ & $8.4(8.8)$ & 0.091 \\
\hline Hospital admissions in the last year, mean (SD) & $0.71(0.8)$ & $1.61(1.7)$ & $<0.001$ \\
\hline Emergency department visits in last 6 months, mean (SD) & $0.39(1.22)$ & $1.21(3.0)$ & $<0.001$ \\
\hline HOSPITAL score, mean (SD) & $3.86(1.4)$ & $4.96(1.8)$ & $<0.001$ \\
\hline LACE index, mean (SD) & $11.31(2.4)$ & $12.59(3.72)$ & $<0.001$ \\
\hline Charlson comorbidity score, mean (SD) & $4.88(3.27)$ & $6(3.78)$ & $<0.001$ \\
\hline Number of discharge medications, mean (SD) & $10.33(6.49)$ & $11.89(6.53)$ & $<0.001$ \\
\hline \multicolumn{4}{|l|}{ Medical comorbidities: } \\
\hline myocardial infarction, n (\%) & $344(26 \%)$ & $150(33 \%)$ & 0.004 \\
\hline congestive heart failure, n ( \% ) & $295(22 \%)$ & $159(35 \%)$ & $<0.001$ \\
\hline peripheral artery disease, n (\%) & $118(9 \%)$ & $45(10 \%)$ & 0.539 \\
\hline stroke, n (\%) & $76(6 \%)$ & $26(6 \%)$ & 0.978 \\
\hline dementia, n (\%) & $40(3 \%)$ & $9(2 \%)$ & 0.239 \\
\hline chronic lung disease, n (\%) & $357(27 \%)$ & $144(32 \%)$ & 0.058 \\
\hline connective tissue disease, $\mathrm{n}(\%)$ & $28(2 \%)$ & $7(2 \%)$ & 0.443 \\
\hline peptic ulcer disease, n (\%) & $59(5 \%)$ & $19(4 \%)$ & 0.797 \\
\hline cirrhosis, n (\%) & $40(3 \%)$ & $25(6 \%)$ & 0.016 \\
\hline diabetes without complications, n ( \%) & $272(21 \%)$ & $127(28 \%)$ & 0.001 \\
\hline diabetes with complications, n ( \%) & $145(11 \%)$ & $88(19 \%)$ & $<0.001$ \\
\hline paralysis, n (\%) & $44(3 \%)$ & $24(5 \%)$ & 0.062 \\
\hline renal disease, $n(\%)$ & $243(18 \%)$ & $142(31 \%)$ & $<0.001$ \\
\hline cancer, n ( \% ) & $97(7 \%)$ & $42(9 \%)$ & 0.194 \\
\hline metastatic cancer, n (\%) & $32(2 \%)$ & $18(4 \%)$ & 0.088 \\
\hline
\end{tabular}

drug treatment regimens are often required for medical conditions such as CHF and diabetes, which were more prevalent in the patients readmitted to the hospital within 30 days of discharge. The higher Charlson scores and a higher prevalence of medication intensive comorbidities such as diabetes in the group readmitted to the hospital within 30 days of discharge is likely to be causal of the polypharmacy. However, only CHF and renal disease appear to be statistically significant predictors of hospital readmission in multivariate analysis.

Interpretation of these findings is complicated because there is no agreement on a standard cut-off point for the number of medications prescribed in the definition of polypharmacy. Problems arise when more medications are prescribed than are clinically required. The concurrent use of multiple drugs or more drugs than medically necessary is polypharmacy. ${ }^{24}$ Various arbitrarily chosen cut-off points are used to evaluate prevalence and 30-day hospital readmission in several studies. ${ }^{25,26}$ Many studies in ambulatory care define polypharmacy as a medication count of five or more medications. The prevalence of polypharmacy in age $>65$ years, the concomitant use of five or more medications, was $51.9 \%$ at hospital admission and $67.0 \%$ at discharge, and it was not associated with a poor hospital outcome. ${ }^{25}$ Often, the hospital readmission predictors include polypharmacy ( $\geq 6$ medicines). ${ }^{26}$

The study that looked at polypharmacy ( $\geq 6$ medicines) showed an estimated odds ratio for readmission for high Charlson scores of 1.7 (95\% CI 1.1-2.6) and of 2.1 for polypharmacy (95\% CI 1.3-3.7). The model yielded a readmission risk estimate of $6 \%$ if neither a high Charlson score nor polypharmacy was present, $9 \%$ if only the Charlson score was high, $12 \%$ if only polypharmacy was present, and $19 \%$ if both were present. ${ }^{26}$

Picker et al identified more than six discharge medications to be independently associated with 30 -day readmission. ${ }^{2}$ The results of our study differ from other published work in the role of polypharmacy, and hospital readmission risk is likely due to significant differences in our study population and other populations. The overall readmission rate ( $27 \%$ vs $21 \%$ ), average age ( 64 vs 55 years), Charlson comorbidity score ( 6 vs 5 ), length of stay ( 8 vs 4 days) and number of medications at time of discharge (12 vs 7) were significantly higher in those readmitted to the hospital when 
Table 4. Multivariate logistic regression of potential risk factors for hospital readmission within $\mathbf{3 0}$ days of discharge

\begin{tabular}{|c|c|c|}
\hline Characteristic & $\begin{array}{l}\text { Odds ratio }(95 \% \\
\text { CI) }\end{array}$ & p value \\
\hline Hospital admissions in the last year & $1.34(1.18-1.52)$ & $<0.001$ \\
\hline $\begin{array}{l}\text { Emergency department visits in } \\
\text { last } 6 \text { months }\end{array}$ & $1.27(1.15-1.40)$ & $<0.001$ \\
\hline HOSPITAL score & $1.45(1.31-1.61)$ & $<0.001$ \\
\hline LACE index & $0.86(0.80-0.93)$ & $<0.001$ \\
\hline Charlson comorbidity score & & 0.340 \\
\hline Number of discharge medications & & 0.225 \\
\hline \multicolumn{3}{|l|}{ Medical comorbidities: } \\
\hline myocardial infarction & & 0.839 \\
\hline congestive heart failure & $1.58(1.18-2.13)$ & 0.002 \\
\hline cirrhosis & & 0.099 \\
\hline diabetes without complications & & 0.416 \\
\hline diabetes with complications & & 0.730 \\
\hline renal disease & $1.36(1.01-1.83)$ & 0.046 \\
\hline
\end{tabular}

compared with the data reported by Picker and colleagues. ${ }^{2}$ Several of these differences (age, Charlson score and length of hospital stay) are known predictors of increased hospital readmission risk and are components of the HOSPITAL score and LACE index. $8,10,17$

The performance of the established and validated HOSPITAL score and LACE index in our study was comparable to prior investigations at this centre ${ }^{27,28}$ and validation studies of the HOSPITAL score ${ }^{11,13}$ and LACE index. ${ }^{13,17}$ Local practice details such as no distinct oncology admitting service and infrequent laboratory testing on the day of hospital discharge likely reduce the accuracy of the HOSPITAL score in this setting. Despite these factors, the predictive ability of the HOSPITAL score was comparable to validation studies of the HOSPTIAL score. ${ }^{11,13}$ Missing laboratory values needed for the calculation of the HOSPITAL score were addressed using the methodology used in the international validation study of the HOSPTIAL score by Donzé and colleagues. ${ }^{11}$

Our study has several important limitations. This study and the international validation study for the HOSPITAL score share an important shortfall by only identifying readmissions within 30 days at the same hospital. ${ }^{11}$ This limitation was overcome in the LACE index original validation study by contacting the patient 30 days after discharge of the hospital to determine the readmission status. ${ }^{17}$ Furthermore, our study is retrospective, single centre, focused on medical patients, small sample size and shaped by local practice patterns (no oncology admitting service, few elective admissions, infrequent laboratory testing on the day of discharge). These local factors could lead to underestimation of the risk of readmission with the HOSPITAL score. The high rate of readmission is likely because the study hospital deals with the underserved high-risk populations in the region. The generalisability of these results may be reduced because of these limitations.

\section{Conclusions}

Our study demonstrated that the number of discharge medications alone does not appear to be a useful tool for a moderate sized community hospital to identify patients at high risk of hospital readmission within 30 days. Other risk scoring systems such as the HOSPITAL score and LACE index are more effective at identifying high-risk patients. Our study further supports the need for more research to explore polypharmacy as a risk predictor for hospital readmission. Evaluating the interaction of specific medical comorbidities and medications may be a highyield avenue for investigation.

\section{References}

1 Jencks SF, Williams MV, Coleman EA. Rehospitalizations among patients in the Medicare fee-for-service program. N Engl J Med 2009;360:1418-28.

2 Picker D, Heard K, Bailey TC et al. The number of discharge medications predicts 30-day hospital readmission: a cohort study. BMC Health Serv Res 2015;15:282.

3 Hasan O, Meltzer DO, Shaykevich SA et al. Hospital readmission in general medicine patients: a prediction model. J Gen Intern Med 2010;25:211-9.

4 Silverstein MD, Qin H, Mercer SQ, Fong J, Haydar Z. Risk factors for 30 -day hospital readmission in patients $\geq 65$ years of age. Proc Bayl Univ Med Cent 2008;21:363-72.

5 Auerbach AD, Kripalani S, Vasilevskis EE et al. Preventability and causes of readmissions in a national cohort of general medicine patients. JAMA Intern Med 2016;176:484-93.

6 Allaudeen N, Schnipper JL, Orav EJ, Wachter RM, Vidyarthi AR. Inability of providers to predict unplanned readmissions. J Gen Intern Med 2011;26:771-6.

7 Zhou H, Della PR, Roberts P, Goh L, Dhaliwal SS. Utility of models to predict 28-day or 30-day unplanned hospital readmissions: an updated systematic review. BMJ Open 2016;6:e011060.

8 Kansagara D, Englander $\mathrm{H}$, Salanitro A et al. Risk prediction models for hospital readmission: a systematic review. JAMA 2011;306:1688-98.

9 Smith DM, Giobbie-Hurder A, Weinberger $M$ et al. Predicting nonelective hospital readmissions: a multi-site study. Department of Veterans Affairs Cooperative Study Group on Primary Care and Readmissions. J Clin Epidemiol 2000;53:1113-8.

10 Donzé J, Aujesky D, Williams D, Schnipper JL. Potentially avoidable 30-day hospital readmissions in medical patients: derivation and validation of a prediction model. JAMA Intern Med 2013;173:632-8.

11 Donzé JD, Williams MV, Robinson EJ et al. International validity of the HOSPITAL score to predict 30-day potentially avoidable hospital readmissions. JAMA Intern Med 2016;176:496-502.

12 Robinson R, Bhattarai M, Hudali T. Vital signs abnormalities on discharge does not predict 30-Day readmission. Clin Med Res 2019: [Epub ahead of print].

13 Garrison GM, Robelia PM, Pecina JL, Dawson NL. Comparing performance of 30-day readmission risk classifiers among hospitalized primary care patients. J Eval Clin Pract 2017;23:524-9.

14 Tong L, Erdmann C, Daldalian M, Li J, Esposito T. Comparison of predictive modeling approaches for 30-day all-cause non-elective readmission risk. BMC Med Res Methodol 2016;16:26.

15 Spiva L, Hand M, VanBrackle L, McVay F. Validation of a predictive model to identify patients at high risk for hospital readmission. J Healthc Qual 2016;38:34-41.

16 Steventon A, Billings ]. Preventing hospital readmissions: the importance of considering 'impactibility,' not just predicted risk. BMJ Qual Saf 2017;26:782-5.

17 van Walraven C, Dhalla IA, Bell C et al. Derivation and validation of an index to predict early death or unplanned readmission after discharge from hospital to the community. CMAJ 2010;182:551-7. 
18 Morath B, Mayer T, Send AFJ et al. Risk factors of adverse health outcomes after hospital discharge modifiable by clinical pharmacist interventions: a review with a systematic approach. $\mathrm{Br}]$ Clin Pharmacol 2017;83:2163-78.

19 Mixon AS, Yeh VM, Simmons S et al. Improving care transitions for hospitalized veterans discharged to skilled nursing facilities: A focus on polypharmacy and geriatric syndrome. Geriatrics (Basel) 2019;4:19.

20 Wimmer BC, Dent E, Bell JS et al. Medication Regimen Complexity and Unplanned Hospital Readmissions in Older People. Ann Pharmacother 2014:48:1120-8.

21 Mansur N, Weiss A, Beloosesky Y. Looking beyond polypharmacy: quantification of medication regimen complexity in the elderly. Am J Geriatr Pharmacother 2012;10:223-9.

22 Morandi A, Bellelli G, Vasilevskis EE et al. Predictors of rehospitalization among elderly patients admitted to a rehabilitation hospital: the role of polypharmacy, functional status, and length of stay. J Am Med Dir Assoc 2013;14:761-7.

23 Lu W-H, Wen Y-W, Chen L-K, Hsiao F-Y. Effect of polypharmacy, potentially inappropriate medications and anticholinergic burden on clinical outcomes: a retrospective cohort study. CMAJ 2015;187:E130-7.
24 Maher RL, Hanlon J, Hajjar ER. Clinical consequences of polypharmacy in elderly. Expert Opin Drug Saf 2014;13:57-65.

25 Nobili A, Licata G, Salerno F et al. Polypharmacy, length of hospital stay, and in-hospital mortality among elderly patients in internal medicine wards. The REPOSI study. Eur ] Clin Pharmacol 2011:67:507-19.

26 Logue E, Smucker W, Regan C. Admission Data Predict High Hospital Readmission Risk. J Am Board Fam Med 2016;29:50-9.

27 Robinson R, Hudali T. The HOSPITAL score and LACE index as predictors of 30 day readmission in a retrospective study at a university-affiliated community hospital. Peer] 2017;5:e3137.

28 Hudali T, Robinson R, Bhattarai M. Reducing 30-day rehospitalization rates using a transition of care clinic model in a single medical center. Adv Med 2017;2017:5132536.

Address for correspondence: Mukul Bhattarai, Internal Medicine Department, Southern Illinois University School of Medicine, 747 N Rutledge St, Springfield, IL 62702, USA. Email: drmukulbhattarai@gmail.com

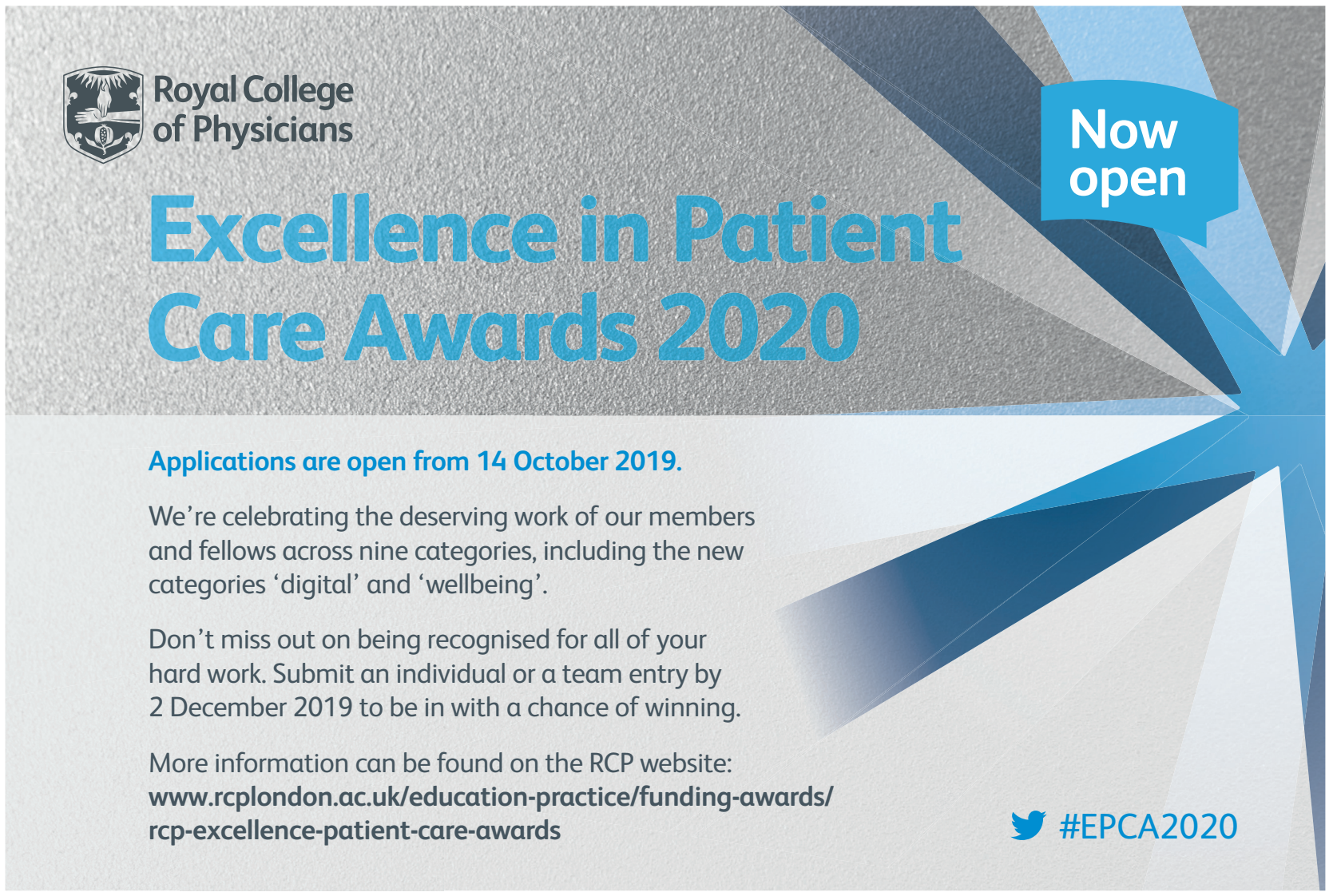

